\title{
Virtual Endoscope System with Force Sensation
}

\author{
Koji IKUTA , Masaki TAKEICHI , Takao NAMIKI \\ Department of Micro System Engineering, School of Engineering, Nagoya University \\ Furocho, Chikusa-ku, Nagoya 464-8603, Japan \\ ikuta@mech.nagoya-u.ac.jp
}

\begin{abstract}
We have developed a Virtual Endoscope System (VES) with force sensation to train inexperienced young doctors and simulate operations that require special technical skills. In this paper, we describe the force simulation mechanism employed by our VES and dynamical models of an endoscope and colon. The force simulation mechanism was developed with the use of four rubber rollers and differential gears. The simple structure of this mechanism enables easy control and stable linear and rotational drive of the endoscope, and we have confirmed its force simulation ability by several experiments. We also developed the dynamical models of an endoscope and colon to calculate reactive force that doctors receive from a colon in real-time, and we confirmed the accuracy of these models by software simulation of endoscopic. We believe this system is adequate for training young doctors in endoscopic insertion.
\end{abstract}

\section{Introduction}

Explosive growth of the elderly population is inevitable in the early 21 st century, and most patients who require colonoscopic diagnosis are aged persons. Although minimally invasive methods for the diagnosis of internal organs have been developed to reduce pain in current clinical settings, with colonoscopy, both discomfort to the patient and the time required for diagnosis heavily depend on the technical skill of the doctor. Moreover, in some cases, inexperienced doctors have damaged colons in colonoscopic diagnosis. Therefore, a training system in colonoscopic diagnosis for young doctors has been desired.

Accordingly, we propose a Virtual Endoscope System (VES) with force sensation for the repeated training of inexperienced young doctors in colonoscopic insertion and operation. Using information on the patient's internal organs, of the system generates a force sensation to simulate the feeling of an actual operation and diagnosis.

\section{Virtual Endoscope System}

The total concept of the Virtual Encoscope System is shown in Fig.1. The VES consists of the following three main parts. 


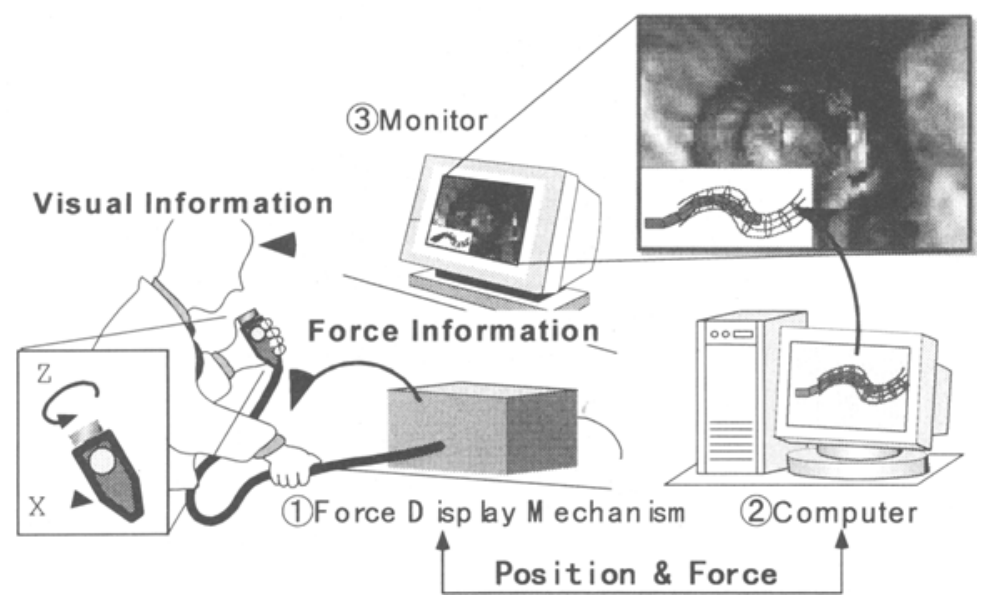

Fig. 1. Virtual Endoscope System with Force Sensation

1) Force Simulation Mechanism: Doctors insert and operate the same type of endoscope used in actual diagnosis.

2) Computer : a high-speed micro computer calculates the reactive force between the endoscope and digestive tube in real time and controls the VES mechanism.

3) Monitor : CT images of the colon are converted into 3D-Computer graphics and displayed on the monitor.

By linking the VES mechanism to the monitor through the computer, the doctor obtains not only visual information but also force sensation according to the position and posture of the endoscope. The insertion of the endoscope in the virtual digestive tube accurately simulates insertion in an actual colon.

The proposed VES has the following features,

1. It can be used with an actual endoscope in various

2. It improves skills through the use of simulated force and visual information

3. It can be used for repeated trainings

4. It can be adapted to simulate any type of data on individual patients or parts

With these features, the system can be used to train inexperienced doctors and simulate operations that require special technical skills.

Force information is a very important aspect in the training of medical skills such as endoscopic operation. However almost all conventional studies have concentrated on the development of visual information from medical image processing, and very few have attempted to simulate the force mechanically. Therefore, in the present study we focused on the realization of a mechanism and a control system for the virtual situation of actual endoscopic operation. 


\section{Force Simulation Mechanism}

\subsection{Basic Concept}

First, the colon and endoscope are modeled in virtual space according to the procedure to be performed by doctors. When the endoscope touches several points of the colon (Fig.2), the basic principle of the dynamics is used to calculate the force at each point as the total force and torque transferred to the doctor's hand. In other words, the force simulation mechanismrecreates the total force and torque and conveys the sensation to the doctor's hand. The force simulation mechanism requires the following specifications:

1. Giving the endoscope two degrees of freedom (infinite linear motion and infinite rotation)

2. Correctly conveying the force and position information calculated in the virtual space to the doctor

To realize these requirements, we developed the mechanism using two components; a "torque-coupled roller mechanism" and "differential gear mechanism."

\subsection{Torque-Coupled Rubber Roller Mechanism}

To consider operation of the endoscope, the force simulation mechanism must give the endoscope "infinite" linear motion and rotation motion. The simplest mechanism is a torque-decoupled type (Fig.3(a)), but this type is seriously flawed by the friction produced by the dormant roller while the other roller drives. Hence, we applied a coupled type (Fig.3(b)) to solve this problem. This type consists two rubber rollers mounted on $45^{\circ}$ axes to the endoscope. By coordinating the rotating directions of the two rollers, we can cancel the opposing frictional forces when we want to drive and transfer thrust and torque to the endoscope.

Though two rubber rollers can realize both linear and rotary drive, for more stable support we use four rubber rollers; two for the linear drive and two for the rotary drive. Therefore, we can control the force simulation mechanism easily.

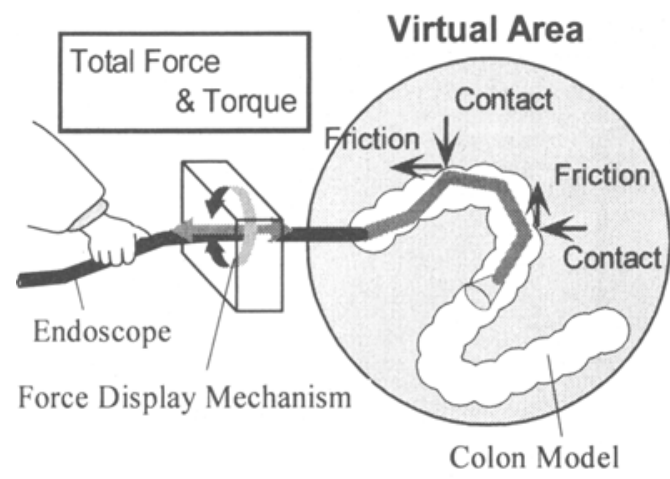

Fig. 2. Concept of Force Simulation System (a)

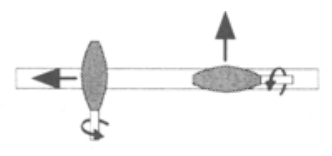

(b)

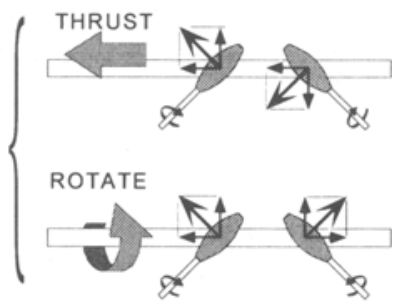

Fig. 3. Two Types of Driving Mechanism 


\subsection{Introducing Differential Gear}

The use of a differential gear offers the following advantages:

1. A reduced number of motors

2. Easier control

3. Reduced friction force between the rollers and endoscope

4. Accurate transfer of thrust and torque to the endoscope

The schematic design of the force simulation mechanism is shown in Fig.4. The rotary drive is realized by the upper unit and the linear drive is realized by the lower unit.

In general, a mechanism using four rollers would require the use of four motors. However, it would be complicated to control coupled-type system through the cooperated use of four motors. For this reason we added two differential gears in this mechanism (shown in Fig.4) to reduce the number of motors from four to two and simplify the control.

In this mechanism, the function of the differential gear transfers the torque of the motor to the tips of both shafts, and the tips of both shafts rotate in the same direction when a motor drives (Fig.5(a)). In this situation, the relation of the rotational velocity between a motor and shafts can be equated as:

$$
2 \omega_{c}=\omega_{a}+\omega_{b}
$$

When the motor is locked (Fig.5(b)), eq.(2) is given because $\omega_{c}$ in eq.(1) is equal to zero.

$$
\omega_{a}=-\omega_{b}
$$

Accordingly, the tips of the two shafts can be freely rotated in opposite directions by the external torque (Fig.5(b)).

For example, when simulating thrust, the motor of the rotary drive unit stays locked, but the two rollers rotate according to the motion of the endoscope by the function of the differential gear. On the other hand, these rollers produce rotational forces to the endoscope. In theory, however, rotary forces have no effect on the endo-

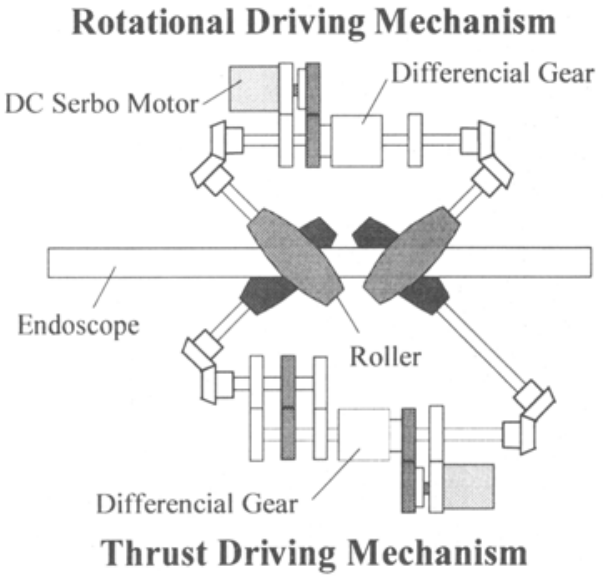

Fig. 4. Concept of Force Simulation Mechanism (a)

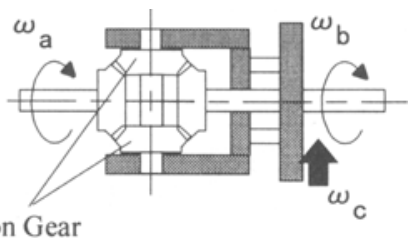

Pinion Gear

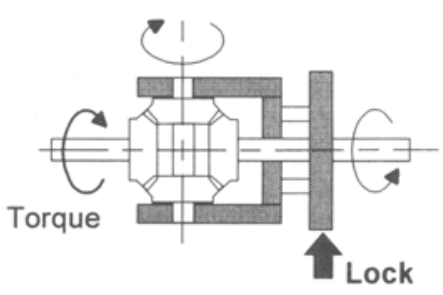

Fig. 5. Basic Motion of Differential Gear 
scope since these forces can be cancelled by the feature of the coupled-type driving mechanism. Thus, with the use of the differential gear, frictional forces are lower than those produced with the decoupled-type.

Finally, we explain the correct transfer of thrust and torque from the rollers to the endoscope. According to the eq.(1), the revolution of the motor is always equal to the total revolution of the shaft tips. Therefore, if the revolution of one roller is reduced some obstacle or problem, the revolution of one of the other rollers is increased by the function of the differential gear, and we can correctly transfer the motor power to the endoscope. In this case, the unnecessary force is transferred to the endoscope because it is impossible to cancel the opposing force against the direction we want to drive. In the present system, however, since we designed this mechanism to realize the linear and rotary drive by each unit, we can cancel the unnecessary force by controlling the motor of the other unit.

\subsection{Force \& Position Sensing}

In this system, we applied a six-component-force sensor for measuring forces of six independent DOFs, and we applied two encoders for measuring the position (linear and rotational) of the endoscope.

Since the system in the present study was desingned for training of endoscope insertion using an actual endoscope, we did not attach any measurement devices to the endoscope. Alternatively, we applied the force sensor beneath the mechanism and placed two encoders on the insertion hole for the endoscope (Fig.6).

\subsection{Adjustable Diameter}

To enable application of various types of endoscopes to this mechanism, we made the spaces between the rollers adjustable. Accordingly, this mechanism can be applied to endoscopes hanging in diameter from 8 to $24 \mathrm{~mm}$, and with a little improvement, it is applicable to training with catheters of $2 \sim 3 \mathrm{~mm}$ in diameter.

\subsection{Prototype (VES-II)}

According to the principles we described above, we developed a prototype model called the VES- $\Pi$. The VES- $\Pi$ is shown in Fig.6 and a close-up view of the rubber
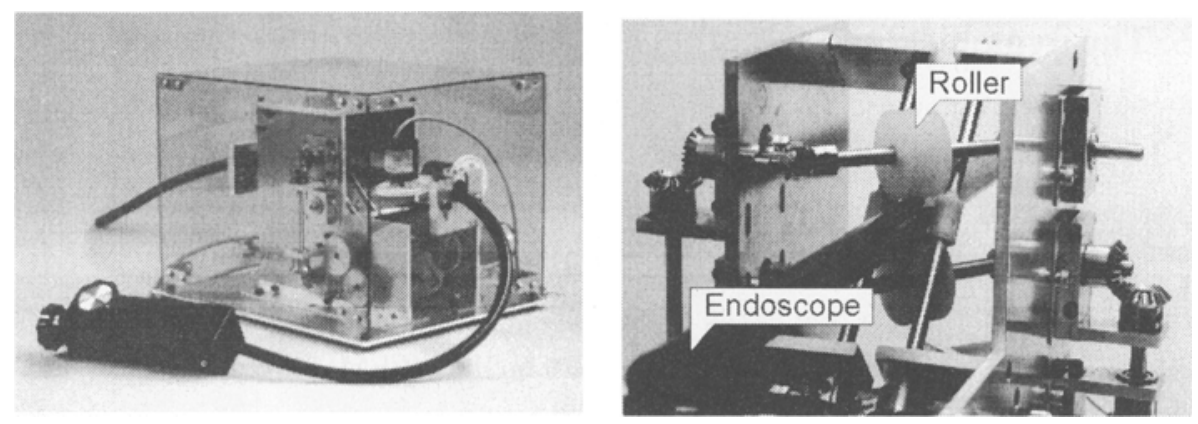

Fig. 6. Force Simulation Mechanism (VES- II ) Fig.7 Close up View of Rubber Roller Mechanism 
roller mechanism is shown in Fig.7. The arrangement of the rollers of the VES- $\Pi$ is slightly different from that of the concept seen in Fig.4. In fact, they are arranged on concentric circles. By locating rollers concentrically, we can drive the endoscope based on the same principle described above but with greater stability.

\section{The Servo System for force simulation}

\subsection{The control method}

First we studied to the realization of a free mode and elastic mode by operating an endoscope. Free mode means a case in which the endoscope does not touch the colon, and elastic mode means a case in which the endoscope touches the colon, which acts against it as a spring.

The control system of the VES-IIgets force information from the force sensor and calculates the value of the theoretical position of the endoscope. Then it compares the value of the theoretical position with the value of the current position measured, and decides what output voltage to supply to the motor.

\subsection{Experiments}

The results of the experiments are shown in Fig.8(a),(b).

In Fig.8(a), we see that the reactive force is almost zero.

In Fig.8(b), the reactive force increases as the deformation becomes larger at each of three types of virtual spring stiffness. This figure shows that the measured reactive force diverges from the theoretical line (solid line). This discrepancy occurred because the static friction between the rollers and endoscope was overcome by the operation force of the endoscope. This problem should be solved by fine tuning of control parameters.

In this experiment, we can feel the sensation of pushing a spring, and we can confirm that the endoscope returns to the beginning position where the endoscope began to touch the virtual colon.

\section{The dynamical models of an endoscope and colon}

\subsection{The presupposition condition}

The purpose of modeling is to analyze the motion of an endoscope in a virtual space, and to calculate the total reactive force and torque which returns from the colon to the hand of the doctor.

The proposed model defines an endoscope as multiple robotic links. Though this analysis is less accurate than the finite element method (FEM), it requires far less time for calculation.

The presupposition conditions for the modeling can be summarized as follows: 


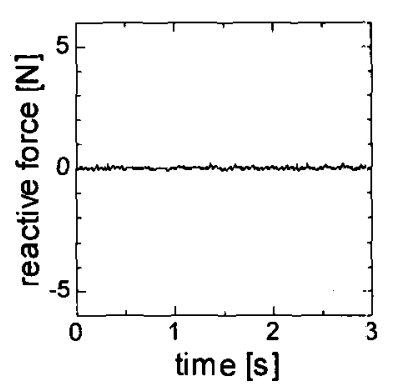

(a)

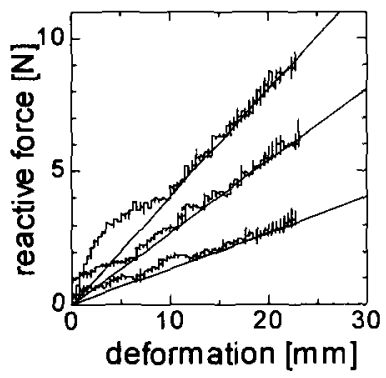

(b)

Fig. 8. Force Simulation Ability of The VES- II

1. An endoscope is defined as multiple links, each comprising a rigid arm combined with viscoelastic components.

2. A colon is defined as a viscoelastic circular cylinder with various diameters.

3. Frictional force between the endoscope and colon is considered using a frictional cone as a representation of the inner contact force.

The algorithm of this modeling is constructed under a motion equation used for the analysis of a manipulator. Considering the actual motion of an endoscope and colon, we attempted to simplify the equation by deducting infinitesimal effect elements.

\subsection{The modeling of an endoscope}

First, we consider a general model of an endoscope and colon which includes all forces of action. An endoscope is clefined as an $n$ multiple links model which has ( $n$ 1) mobile joints with viscoelastic components. Since a real endoscope is flexible, we consider an endoscope to have the infinite joints. The motion of endoscope is limited by the number of joints. Thus, we must decide the number of joints and the length of one link. As the number of joints increases, the accuracy of the calculated motion improves. The force simulation mechanism requires continuous real-time analysis in virtual space. In other words, it is necessary to compromise and approximate in the range where the doctor can recognize.

Therefore we must find a suitable number of joints to compromise on processing time. Since the direction of the whole endoscope is determined by the displacement and frequency of the tip, the assumption of modeling in the tip is very important. Modeling in the root part is not a severe problem since the motion is very slow. Therefore, our design, the length of each link gradually increases from the tip to the root.

Next we define the degree of freedom of each mobile joint. The joint nearest to the hand has a thrusting and rotating mechanism and the other joints have a rotating mechanism in two rectangular directions. Each joint has a viscoelastic component (Fig.9).

Here we derive an equation to formulate this system. Multiple links can be expressed using Newton-Euler's equation of motion as follows.

$$
\begin{gathered}
\mathbf{M}(\mathbf{q}) \ddot{\mathbf{q}}+\mathbf{V}(\mathbf{q}, \dot{\mathbf{q}})+\mathbf{G}(\mathbf{q})=\sum_{i}^{n} \mathbf{J}_{\mathbf{r}_{i}}^{T}(\mathbf{q}) \mathbf{F}_{i}+\mathbf{T} \\
\mathbf{T}=-K\left[\mathbf{q}-\mathbf{q}_{0}\right]-D \dot{\mathbf{q}}
\end{gathered}
$$


$\mathbf{M}(\mathbf{q})$ means the inertia matrix. $\mathbf{V}(\mathbf{q}, \dot{\mathbf{q}})$ means the centrifugal force, Coriolis force. G(q) means weight function. Here we explain the first function on the right side of eq.(3). Contact force occurs at $n$ points where the colon wall contacts each mobile joint and the tip of the endoscope. Then contact force vector $\mathbf{F}_{i}$ is converted into the force of each joint in local coordinates using Jacobian matrix $\mathbf{J}(\mathbf{q})$ systems. Functional force $\mathbf{T}$ of eq.(3) is defined as the sum of the elastic force (elasticity coefficient $K$ ) and viscous force (viscosity coefficient $D$ ) of a mobile joint. $\mathbf{q}_{0}$ means the position of the balance of each joint in local coordinates.

\subsection{The modeling of a colon}

The shape of the colon is defined as a viscoelastic circular cylinder with various diameters. Here we consider the following equation:

$$
\begin{aligned}
& S(\mathbf{r})=0 \\
& R=\phi(s)
\end{aligned}
$$

Curve $\mathrm{S}$ expresses the shape as the central curve of the colon model (Fig.10).

Setting radius $R$ according to $\mathrm{S}$, we express the three-dimensional shape of the colon with various diameters. These equations are able to correspond to deformation of the colon according to contact force. Moreover, we consider the elasticity coefficient $K_{d}$ and viscosity coefficient $D_{d}$ of the surface of the colon wall. The static friction coefficient $\mu_{s}$ and kinetic friction coefficient $\mu_{d}$ are also considered.

\subsection{Simplification of model equation}

Here we explain the reduction of the terms of the above equations.

First, the deformation speed during insertion of the endoscope is very slow. Thus, the terms $\mathbf{M}(\mathbf{q})$ and $\mathbf{V}(\mathbf{q}, \dot{\mathbf{q}})$ in eq.(3) are thought to be sufficiently smaller than the weight of the endoscope or contact force.

Therefore, eq.(3) can be simplified by reducing the terms $\mathbf{M}(\mathbf{q})$ and $\mathbf{V}(\mathbf{q}, \dot{\mathbf{q}})$ as follows:

$$
\mathbf{G}(\mathbf{q})=\sum_{i}^{n} \mathbf{J}_{\mathbf{r}_{i}}^{T}(\mathbf{q}) \mathbf{F}_{i}-K\left[\mathbf{q}-\mathbf{q}_{0}\right]-D \dot{\mathbf{q}}
$$

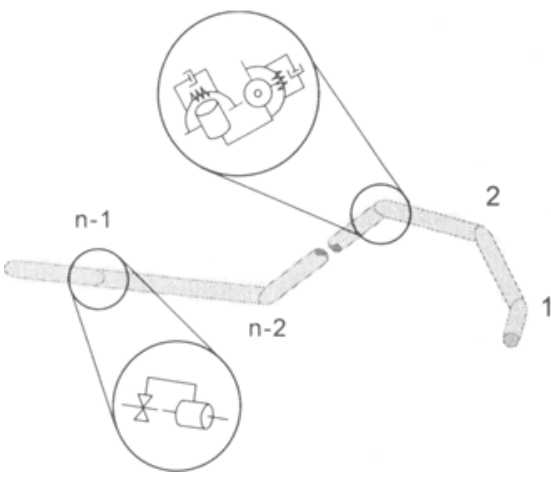

Fig. 9. Endoscope Model

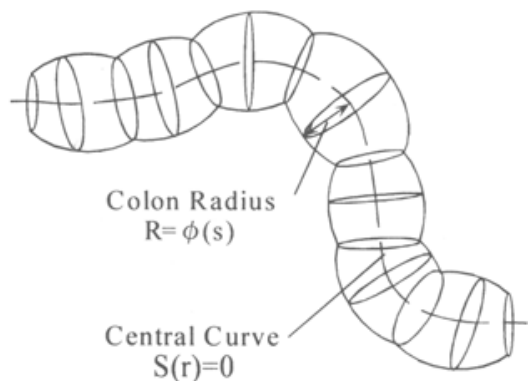

Fig. 10. Colon Model 
By solving this equation of motion on the short time-interval, the dynamic behavior of the endoscope can be analyzed quasi-dynamically.

The viscous term cannot be omitted to keep a joint angle of each mobile joint. If omitted, the joint angle returns to the previous position of balance at the next moment the contact force is absent.

\subsection{Calculation of contact judgment and the contact force}

Here we explain the contact judgement between the endoscope and colon.

First, we can calculate the distance between a joint of the endoscope and the central curve of the colon. Then, the geometry of the contact point can be estimated for comparison with the radius $R$ of the colon model.

In the case of contact, the contact force is expressed as follows in the normal coordinates:

$$
\mathbf{f}_{i}=\left\{-K_{d}\left(l_{i}-R\right)-D_{d}\left\|\mathbf{v}_{i}\right\|\right\} \cdot \frac{\mathbf{v}_{i}}{\left\|\mathbf{v}_{i}\right\|}
$$

where $l_{i}$ means the distance between the joint and nearest point of the central curve, and $\mathbf{v}_{i}$ means the velocity vector of a joint in the normal coordinate.

Next, $f_{i}$ is divided into vertical and tangential components of the colon on contact points to examine the frictional condition. Supposing vertical vector as $\mathbf{n}_{i}$, the vertical component of the contact force $\mathbf{f}_{n i}$ and tangential component $\mathbf{f}_{t i}$ are given as follows:

$$
\mathbf{f}_{n i}=\mathbf{f}_{i} \cdot \mathbf{n}_{i} \quad, \quad \mathbf{f}_{i i}=\mathbf{f}_{i}-\mathbf{f}_{n i}
$$

From these vectors, the frictional condition can be judged.

Here, when the static frictional condition of the endoscope, reactive force $F_{i}$ is expressed by of following:

$$
\mathbf{F}_{i}=\boldsymbol{f}_{i}
$$

Then the kinetic frictional condition, $F_{i}$ is expressed by

$$
\mathbf{F}_{i}=\mu_{a}\left\|\mathbf{f}_{n i}\right\| \cdot \frac{\mathbf{f}_{t i}}{\left\|\mathbf{f}_{i i}\right\|}+\mathbf{f}_{n i}
$$

The difference of each condition of use of the frictional cone is indicated in Fig.11(a), (b), respectively.

After transforming the reactive force in the normal coordinates to one in the local coordinates of each mobile joint by the transform matrix, it is substituted into the equations of motion of the endoscope.

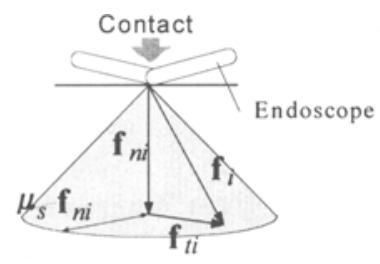

(a)static frictional condition

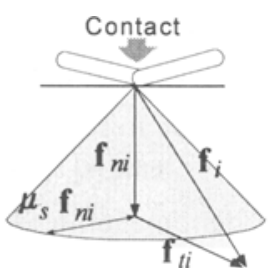

(b)kinetic frictional condition

Fig. 11. Frictional Cone 


\subsection{Simulation}

We have analyzed the above dynamical models of the endoscope and colon.

Here the endoscope is defined to have five mobile joints. The first joint has a thrusting and rotating mechanism and the other four joints have rotating mechanisms with two degrees of freedom.

We established the central curve of the colon as the shape of the cubical parabola equation according to eq.(9). Then we formed the colon by locating various diameters along the central curve. In this model, the central curve is not deformed but the diameter of the colon is easily deformed, in order to simulate the viscoelastical property of the colon wall.

In this paper, we decided our parameters (e.g. the elasticity and viscosity coefficients of the dynamical models) in accordance with the features of the force simulation mechanism. In actuality, however we would like to base our decision on these parameters on the propeties measured from actual endoscopes and organs in the next step.

The result of simulation is shown in Fig.12. These graphs indicate the result obtained for an endoscope inserted by constant thrusting force. Fig.12(b) shows the variation of position and Fig.12(c) the variation of reactive force.
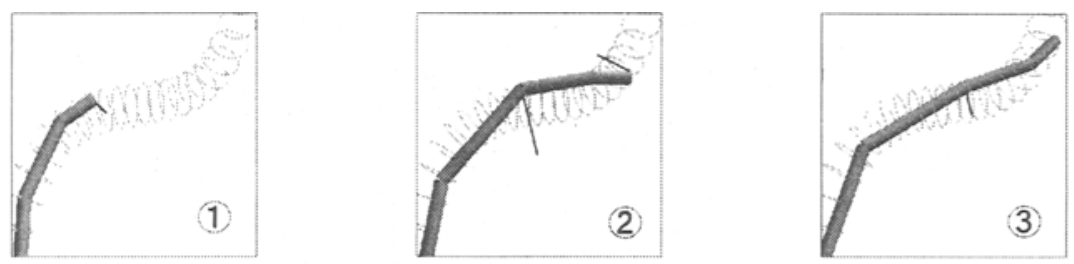

(a) graphical Interface

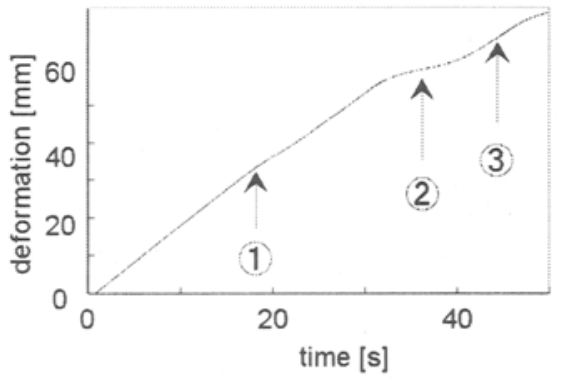

(b) defromation-time

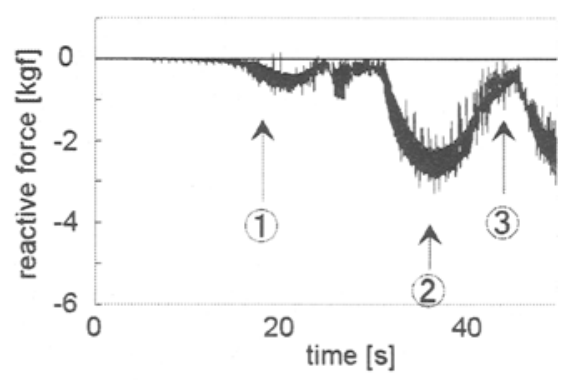

(c) reactive force-time

Fig. 12. The Result of Simulation 
Although the input thrusting force is constant, the velocity of the tip, a time differential of displacement, varies between $30 \mathrm{sec}$ and $40 \mathrm{sec}$ in Fig.12(b). This behavior occurs because the endoscope is subjected to substantial reactive force when it contacts colon wall, where the tip must change the direction according to the cubical parabola equation. However, after turning the tip to the direction along the central curve at 40sec, the reactive force decreases (Fig.12(c)).

Fig.12(a) shows the graphical interface viewed by the doctor during the procedure. The thick line represents the endoscope and the successive circles depict shape of the colon. The straight thin lines spreading from each joint are the reactive force vectors. The size and direction of force can be visually confirmed.

These results confirm that an endoscope insertion operation could be qualitatively realized using an endoscope and colon model. The simplification of eq.(3) did not influence the reality.

\section{Total control system with force sensation}

We realized a total system that conveyed to a doctor the actual sensation of inserting an endoscope to the colon by linking a force simulation mechanism (VES-II) with a dynamic model and monitor display connected via a high-speed micro computer (Fig.13).

Since the total system can be downsized, a compact desktop training system will be available for general hospitals in the near future.

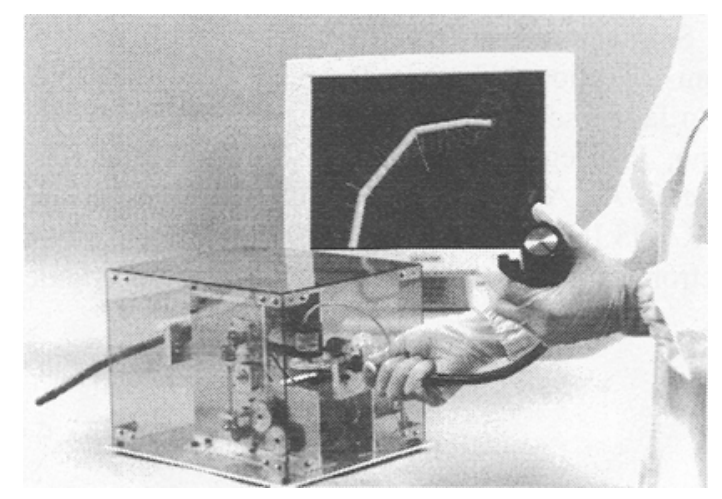

Fig. 13. Total Virtual Endoscope System and Monitor Display 


\section{Conclusions}

We proposed a virtual endoscope system (VES) with force feedback and sensation, and we developed a force simulation mechanism to realize a training system for colonoscopic diagnosis in a virtual digestive tube. It can convey the sensation of reactive force from a digestive tube during insertion.

The dynamical models of both the endoscope and digestive tube were formulated in real-time to calculate the interactional force between them. The force simulation ability of the VES was successfully verified by several experiments.

\section{Acknowledgement}

The authors would like to specially thank Dr. Fumio Isaji for his useful advice, as well as Mr. Takahashi, Mr. Senda, Mr. Nakanishi, and Mr. Tachibana for their support during the making of this equipment.

\section{References}

1. Koji Ikuta, Takao Namiki, Masaki Takeichi: "Study on Virtual Endoscope System with Force Sensation", Proc. of 2nd Annual Conference of the Robotics Symposia, pp.19-22 1997 (in Japanese)

2. Koji Ikuta, Takao Namiki, Masaki Takeichi: "Study on Virtual Endoscope System with Force Sensation -2nd:Verfication of force sensation and Mathematical model", Proc. of 15th Annual Conference of the Robotics Society of Japan, pp.19-22 1997 (in Japanese)

3. Shigeo Hirose, Shinichi Amano : The VUTON: High Payload High EfficiencyHolonomic Omni-Directional Vehicle, Proc. Int. Symp. on Robotics Research, pp. 253-260 1993 (in Japanese)

4. K.Mori, A.Urano, J.Hasegawa, J.Toriwaki, H.Annno and K.Ktatada: Virtualized Endoscope System -An Application of Virtual Reality Technology to Diagnostic Aid- ,IEICE* TRANS.INF.\&SYST., Vol.E79-D, No.6, pp.809-819, 1996.(*IEICEInstitute of Electronics, Information and Communication Engineering of Japan) 